\title{
Strategies in Knowledge Treatment Decision-Making and Operating Conceptual Research
}

\author{
Almada $\mathbf{F}^{1}$, Fernando $\mathrm{C}^{2}$ and António Vicente ${ }^{3 *}$ \\ ${ }^{1}$ Retired University Professor / Independent Researcher, Portugal \\ ${ }^{2}$ University of Madeira - CITUR, Portugal \\ ${ }^{3}$ University of Beira Interior - CIDESD, Rua Marquês D'Ávila e Bolama, 6200-001 Covilhã, Portugal \\ *Corresponding author: António Vicente, University of Beira Interior-CIDESD, Rua Marquês D’Ávila e Bolama, 6200-001 Covilhã, \\ Portugal
}

\section{ARTICLE INFO}

Received: 幽 November 21, 2020

Published: 幽 November 25, 2020

Citation: Almada F, Fernando C, António Vicente. Strategies in Knowledge Treatment Decision-Making and Operating Conceptual Research. Biomed J Sci \& Tech Res 32(2)-2020. BJSTR. MS.ID.005212.

\begin{abstract}
The changes we experience can hardly be efficiently accomplished without structural changes and the operationalization of tools that perform the rupture (Thomas Kuhn). The treatment of knowledge in this new frame of reference, although taking advantage of many instruments that come from the past, requires a functional integration of processes and the establishment of dialectics that were simply ignored.
\end{abstract}

\section{Introduction}

Planning, having a project, defining a course... in addition to: 1 ) allowing decisions to be made in advance and organizing in order to increase the probabilities and achieve what we intend to give, 2) the possibility of having (a priori and a posteriori) the means that facilitate the identification of mistakes made. But to plan we must have a model, a map, that: 3 ) allows us to define the path to follow and the possible objectives, from where we can, 4) define the advantages and disadvantages of each one and distinguish and choose the one we want. Organizing, although it has costs (there is nothing that does not have them), in addition to the four possibilities identified above, it also has, what we still tend to ignore, two more advantages: 5) the training of programming without "having to leave", which increases our capacities to structure and configure similar processes, without the costs and risks inherent in facing the situation (which does not dispense doing it in time), and, 6) the pleasure of dreaming about the project and the living in "virtual reality", which is certainly another benefit of enormous value, which sometimes even replaces what we mentioned in 1) and 3).
But in spite of all these possibilities that, if well explored, will be advantages, the feeling that we may have to ignore all this and have neither a plan, nor a model, nor objectives, nor an organization... is that we have the benefit that we are never mistaken, because fortune will always take us anywhere and that, having not thought about alternatives, with a little optimism, we will always be in the best of worlds. In this way, we can feed our certainties, our ability to be infallible (for if we are never wrong!) and, therefore, we can benefit from all the other advantages of ignorance. Which, with some restriction in the use of intelligence, allows us to exercise other characteristics that are the prerogative of nescience, such as insolence, impertinence, shamelessness, daring ... (an evil never comes alone). At the individual level, there may even be no perception of the costs (often very high) for the individual (which we can almost consider to be not serious, as he will not even be aware of it) and for others.

In this way, we have an alternative between the possibility of asking ourselves questions, looking for the best solutions 
and trying the best chances of success, with all the anxieties and doubts that will not fail to arise, despite the security that we will thus win, or the reverse solution of simply (?) ignoring the risks, which will not cease to exist, the possible advantages that can be achieved, and the pleasure of the path and its challenges that, many times (fortunately) will not be unimportant. The organism itself in extreme and unbearable situations, makes choices and follows similar strategies when it follows hierarchical solutions in the following way, unconsciousness, coma, like that which will naturally follow death unless appropriate measures are taken. Continuing the analysis of this process, we cannot (at least do not want to) fail to mention, in addition to the direct effects, there are still a number of other consequences, let us call them collateral, even less evident, but, perhaps, more serious due to the continued effects that it has, even in the long run.

The destruction of the gains that could have been obtained with the invested work demoralizes, that is, it removes the will to perform and the pleasure of doing and of achieving, which are, we think, essential in this whole process. The usual lame excuse of "it was bad luck, next time we'll have better luck" does not stick. Over time these procedures erode competitiveness and even lead, with the chain of excuses and unreasonable justifications (as well as envy, greed, jealousy, etc.), which are the result not only of a deficient human formation, but also of this type of processes) that make it possible to survive the desolations, a discourse (and its practice) and a self-destructive conceptual structure of which it is not difficult to find examples in many places, institutions and contexts. [Note: the term competitiveness that we expressly and intentionally use above is a good indicator to identify places, institutions and contexts in which the described process is usually easy to recognize].

Now, this situation is much more criticizable in science, (in spite of the many very serious distortions, per se and the consequences they have, which are verified) where professionals "are paid to think", contrary to what they are still in the majority of other professions where the payments are, mainly, to carry out the performance of predetermined algorithms, more or less open, more or less defined. However, very quickly, because these are functions that can be performed by automated or robotic systems and with lower costs and problems, human labor will give way to informatized production, with the resulting consequences.

\section{The Standard Model Knowledge Treatment Strategies}

We are not referring to a standardized model understood as a standard model, a "unique" and generalizable model, of course. When we increase the precision considered, and the inherent rigor, and, consequently, the capacity for discrimination grows, we start to have a situation in which "each case is a different case", admitting the existence of a unified model generalizable to all situations that we could deal with, it would be completely unforgivable.
For the most part, in science, where the level of detail already reached, in most areas of knowledge can be considered as such [Note: differentiate between the area of knowledge, the body of knowledge, and the problem related to an issue, or be the set of answers to the questions that can be asked in relation to a given subject], a level where it can be distinguished, thanks to material and conceptual instruments is enormous [Note: compared to what was possible for us only a few years ago, but not in relation to a real ability to understand and explain the issues that arise, an area in which we only scratch what's going on, as, for example, "artificial intelligence" alerts us], thinking about a "single solution" would not be suitable.

Let us think, then, of a standard model as an example, principle, structure, reference, which allows us to debate, in this case, the strategies of knowledge, decision-making and the use of the tool "structured knowledge" (ie science) in the operationalization, whether research, or in the different forms in which it intervenes in the construction of "work" (conceptual or material, in support of development, technology, and other forms of application that have the foundations and foundations in science). In this way, we will be able to discuss the guidelines to be considered, the precautions to be taken, the pitfalls we should not fall into, the aspects to be privileged, the explorations we should try to carry out, the scales appropriate to the problems faced, etc. Then (even only afterwards if we want to be efficient in using the tool - a tool that is not used episodically, a situation in which anything can perform the function in an expeditious way, but in a situation where frequent use of the tool advises for optimization and consequent specialization) understanding the function and the functionality, we can establish the cost / benefit ratio that we intend to accept for the objectives sought in the existing context, and, therefore, the knowledge strategies that we will use, the conditioning of the decision making that we will perform and the operational framework that we are going to do.

We live in times of change, but we usually make the change without realizing its consequences and effects. What will be natural if our conception starts from a fact for its analysis and from there, we draw the consequences that this fact has. "A fact is a fact", Karl Popper told us, but, argued Thomas Kuhn, "the interpretation of the fact depends on the paradigm in which we are", giving importance to the frame of reference we use. However, we hardly understand what is a fact through the mere analysis of its parts, even if this analysis is profound and care is taken to make a synthesis of what we were able to define in the analysis made. On the other hand, it is often the case that understanding a fact results from the consequences and effects it has. Even if the fact is a mechanism in which the parts are identifiable and the intention behind it is known. "Reverse engineering" often follows this process. But if the fact is something more complex, with components studied by different areas of knowledge and if even, what is normal, we are 
unaware or it is difficult to identify some of its components, the possibility of understanding, and even more of explaining "the fact", it is difficult to accomplish. Karl Popper overcame this difficulty with the "conjecture and refutation" proposal.

Thomas Kuhn did something similar (in terms of the strategy used) with the sequence “... crisis » rupture » new science ...", generating a new paradigm capable of responding "better" to the problems to be dealt with.

What is always sought, in the end, is to find more effective coherences, outdated that is the search for the truth (which only dogmas allow to consider, but which in science is a mere remnant of past times and / or adaptations that were not made to the evolution of knowledge). Coherences that are expressed in models, conjectures, representations that, at different levels (everything has a cost and the precision and the ability to discriminate pay for themselves) and sometimes there are costs that are not justified for the benefits or uses that we want. [Note: the need for this balance between the costs and the intended benefits is a constant in the whole research / development / technology / marketing (RDT\&M), which follows the evolution of any product, material or conceptual, from conception to consumer and even in its use.

Another coherence that we should not forget even when we treat only one of its parts, such as, for example, the investigation to support the process, as there are investigations that do not justify the costs that they have in some evolution phases - let this not be confused situation with the investigation that was once called fundamental research]. Multiple ruptures (Kuhn's concept) were necessary to reach where we are today, as well as competition and the confrontation of models so that we can define the solutions (the tools) best suited to the purposes in view. [Note 1: Let us not forget that the search for truth is left behind, because it is incongruous. Note 2: Science, that is, the understanding and explanation of phenomena, which are increasingly complex and whose understanding is less and less intuitive, in a coherent and ... refutable way (Popper would say), is not a product "just for scientists", as this would lead to: a) supporting the promotion of dogmas through the dissemination of partial aspects or out-ofcontext conclusions; b) had difficulty in financing; c) was subject to distortions and / or manipulations and / or exploitation with consequences that can be serious.

These determinations imply and demand a (difficult) disclosure made so that there is an understanding of the science phenomenon, at the possible level, but at a necessary level too, before they send "the mad scientists" to "the guillotine" or, what would not be best solution, that everyone feels entitled to build conjectures that, without capable foundations, so not refutable, are launched (today communication is easy) on social networks generating "sources of demagogy", discrediting the entire scientific process... or even worst...]

\section{Create Models Knowledge Treatment Strategies}

In a succinct and basic way we assume that a model is built from a set of assumptions considered legitimate by those who build it (if they are false, they will then be refuted, rejecting the created model), constituting axioms, postulates, etc., that do not have to be demonstrated (otherwise we would always be "backwards" and we couldn't move forward), which serve as a foundation on which a set of premises is built so that a syllogism can be drawn, a conclusion, just as in a addition we have plots that, once together, give a result. A conclusion that is not the "truth" (we insist), but a way that allows us, in the end, to put the classic q.e.d. (that is, quod erat desmonstrandum-which we were trying to demonstrate - naturally within the restricted framework of the parameters presented, and for this reason it is not "the truth"). We have a "starting point" to begin a debate. It is not the end of the process. There was someone who exposed himself presenting a position that he thinks coherent, with the foundations (postulates...) on which he rests, the parameters he considers important (the premises), and a way of articulating them to draw a conclusion (a syllogisms). What makes the whole, the model presented, refutable, if it does not present a global coherence, that is, if any of its components is false.

In this way we can refute considering, namely:

a) Support postulates: Are they over? Missing? They are wrong? ...

b) The premises: Are they right? Wrong? Poorly articulated? Aren't they enough? ...

c) Will the conclusion be different with other parameters? Is it badly taken? ...

d) What other errors are pointed out? What justifications do we have? ...

e) What other models can be presented as an alternative? ...

The discussion is no longer based on "I think ...", "I believe ...", "my opinion is that ..." The debate ceased to be a confrontation of personal positions and became a dialogue of reasons. There has been a profound transformation because, with the model: 1) we have moved from a world of absolutes, personal convictions, to a world in which reasons, coherences / dialectics are exchanged; 2) we have passed from the opposition of people, to a confrontation of ideas, we stopped referencing ourselves in relation to an external standard (a truth sometimes made in different contexts), so that each of the participants could refine their frame of reference. Therefore, we will have that the models can (should) evolve because little by little they are limiting flaws and refining suggestions, creating consensus and divergences that are the basis for launching new investigations. 


\section{Advantages of Having Models Knowledge Treatment Strategies}

The models in the knowledge treatment strategies, which we necessarily deal with here briefly and succinctly, are nothing more than representations that must be conceived according to the existing capacities and the purposes for which they are intended, that is, as already mentioned above, a plan, have a project, set a course...

In this way, we have models in which aspects considered to be dominant for the function to be performed are privileged. In the knowledge treatment strategies, we have models, such as:

a) Descriptive Models: which list the components.

b) Analytical Models: which reference the parts and, eventually, their articulations.

c) Functional Models: which describe the possible dynamics.

d) Models...

Descriptive and analytical models had a great predominance and were essential to know what existed and we could have, before leaving for the realization of a work. Today this information is easily available and at very low costs (financial, time, etc.), on computer media that, in addition to being economically accessible, also have the enormous advantage of being permanently updated, a service that, in most cases, was only bearable by large and consequently heavy organizations. This process has been streamlined for users of the service, but also for the structure that maintains the cataloging and inventory of the means to which we can have access (tools and utensils, conceptual or materials, basic equipment, components of the most varied types, parts, books and documents, etc.), which often found ways to be paid indirectly so we can give priority to aspects of functionality, at the factory, school, university, company, etc.. Thus, functional models are now in greater demand and more important, so we will now present some practical situations of interest and ways of application. Examples of the use of functional models in Knowledge Treatment Strategies and in Decision Making and Operationalization:

\section{In Research}

The model, by requiring global coherence, allows the identification of anomalies, points that must be investigated because they appear to be possible flaws. Flaws that are already integrated in the global framework of the model (which in this case could be a theory), which allows, without major concerns and investments in this regard, a continuity that facilitates transdisciplinary and the assimilation of new knowledge that may be produced by this process, in the initial model or even the change to another model that will replace the previous one (in a process of continuity and complementarity, therefore, and not in a change that will later require articulation with the existing framework).

\section{In Higher Education}

Since education / human formation in general, and most of all in higher education, the dominance of the transmission is no longer applicable of already structured knowledge (the predominance of the use of descriptive and analytical models), that is, the teaching of knowledge already created and is widely available in the most varied supports (books, films, magazines, etc.), the transition to a dominance of learning for the use of functional models allows to make a break with the past and the profitability of the new available resources. In the eternal debate university versus polytechnic institutes, if we consider the privileged models in each of these types of institutions, the distinction becomes clear, since universities will preferentially use "explanatory models" and polytechnic institutes "application models". Knowledge remains in both types of institutions profound and well-founded, but applied, preferably, in different contexts.

\section{In Education}

It is certainly not at the level of higher education that the use of models (more applied or more conceptual) will begin. Once the transmission of knowledge has been solved with the use of what is available in the most diverse supports, the function of the teachers becomes the dynamization of the use of models (as it begins to be done in some experimental situations, using, often, computers and robotization as a means of motivation) and support and advice to the work developed by the students, not only as a way to create the domain of useful dynamics, but also as a way to contextualize the acquisition of knowledge that will be selected and fixed in this way and that they will not depend on external programs that facilitate the appearance of biased ways of reaching them (the typical example of the "book that contains what is necessary for the exam"). Thus, the process of searching for the necessary knowledge (which is already done in the most diverse fields, but especially in the less institutionalized ones) benefits, making the process of decorating content an implicit side aspect.

\section{In Adapting to New Conceptual Frameworks}

When it is intended, for example, to move to a conceptual framework according to Einstein's proposal to consider the phenomenon not as an independent and isolated factor, but integrated in the triple relation phenomenon / signal / observer, it is enough to integrate in the framework in which above we briefly expose the globality of postulates / premises / syllogisms and the dynamics of their articulation (a model of knowledge), similar models of the sign and the observer, and, considering the new dialectics that have come to be established, broaden the scope of what is considered and start to have a new globality for the rupture to take effect. 


\section{Conclusion}

In a proposal that for the less attentive may seem to be a mere rearrangement of the past, we warn of aspects of detail that, although apparently innocuous, operationalize a rupture in the treatment of knowledge and in the strategies used in this process. Furthermore than implementing in this way the unification of the fields in which knowledge is treated and developed, at the same time the aim is to achieve independence that allows different paths and

\section{ISSN: 2574-1241}

DOI: $10.26717 / B J S T R .2020 .32 .005212$

António Vicente. Biomed J Sci \& Tech Res

CC (i) This work is licensed under Creative

Submission Link: https://biomedres.us/submit-manuscript.php different methodologies to be followed, without being lost, on the contrary gaining at the level of precision and rigor, as the increase in discrimination increases the depth with which problems are dealt with. In this sense, the use of models, if the necessary forms and safeguards are taken, will enable us to identify anomalies that alert to the corrections needed to be made and the research work to be carried out so that some open spaces previously ignored can be considered and an overall coherence can be achieved.

$\begin{array}{ll}\text { BIOMEDICAL } & \text { Assets of Publishing with us } \\ \text { RESEARCHES } & \text { - Global archiving of articles } \\ & \text { - Immediate, unrestricted online access } \\ & \text { - Rigorous Peer Review Process } \\ & \text { - Authors Retain Copyrights } \\ \end{array}$

